\title{
Constructing a Research Grade Interactive Remote Microscopy Application-Goals, Challenges, and Results
}

\author{
Don Appleman*, Glenn Fried*, Benjamin Grosser*, Daniel Weber*, Chas Conway*, and Scott
} Robinson*

* Imaging Technology Group, Beckman Institute, University of Illinois, 405 North Mathews Avenue, Urbana, IL 61801

The Bugscope project, an educational outreach program for K-12 classrooms, provides an opportunity for students and teachers to remotely operate a scanning electron microscope to image "bugs" at high magnification. The microscope is remotely observed [Fig. 1] and controlled [Fig. 2] in real time from a classroom computer over the Internet using a web browser. In addition to the user interface, the Interactive Remote Microscopy Application (IRMA) software features interactive chat among all participants, automated data archiving and session administration, a communications layer, and server software.

The initial implementation provided still images from the microscope, a reduced set of controls (i.e. focus, contrast, magnification, etc.), and simple administration, scheduling, and data archiving functionality $[1,2]$. Although this worked well for educational outreach purposes, it is insufficient for serious research. In particular, image latency made Bugscope difficult to use for focus operations and navigation of the stage.

Beyond the educational outreach opportunity, Bugscope is intended as a proof-of-concept venture for a number of desired capabilities. With sufficient control of the microscope and a true to life viewing experience, remote microscopy can be proven valuable for remote and collaborative research among isolated researchers, training new users in the operation of the microscope, and the teaching of classes ranging from nanoscience to biology. The introduction of low-latency streaming video to Bugscope, combined with full control over all microscope parameters, and automated administration and session preparation, addresses these goals.

This paper focuses primarily on the goals targeted by the addition of streaming video, the technical challenges (and their solutions) that were encountered along the way, and the results found when the new version of the software was deployed. Specific challenges in this effort included video latency, video frame rate, image size, and image quality, plus management of available bandwidth. This paper outlines in detail the tradeoffs involved, the options available, and the tools and solutions chosen to address each need. Results indicate that, for some classes of users, the IRMA application is ready for use in research-grade science [3].

\section{References}

[1] C. S. Potter et al, Proceedings Microscopy and Microanalysis 1999, pp.514-515.

[2] C. S. Potter et al, Microscopy and Microanalysis, Volume 7, Issue 03, May 2001, pp249-252.

[3] Development work on this project is funded by a grant from the National Aeronautics and Space Administration. The pre-existing work of Nick Kisseberth and Ben Grosser is gratefully acknowledged. 


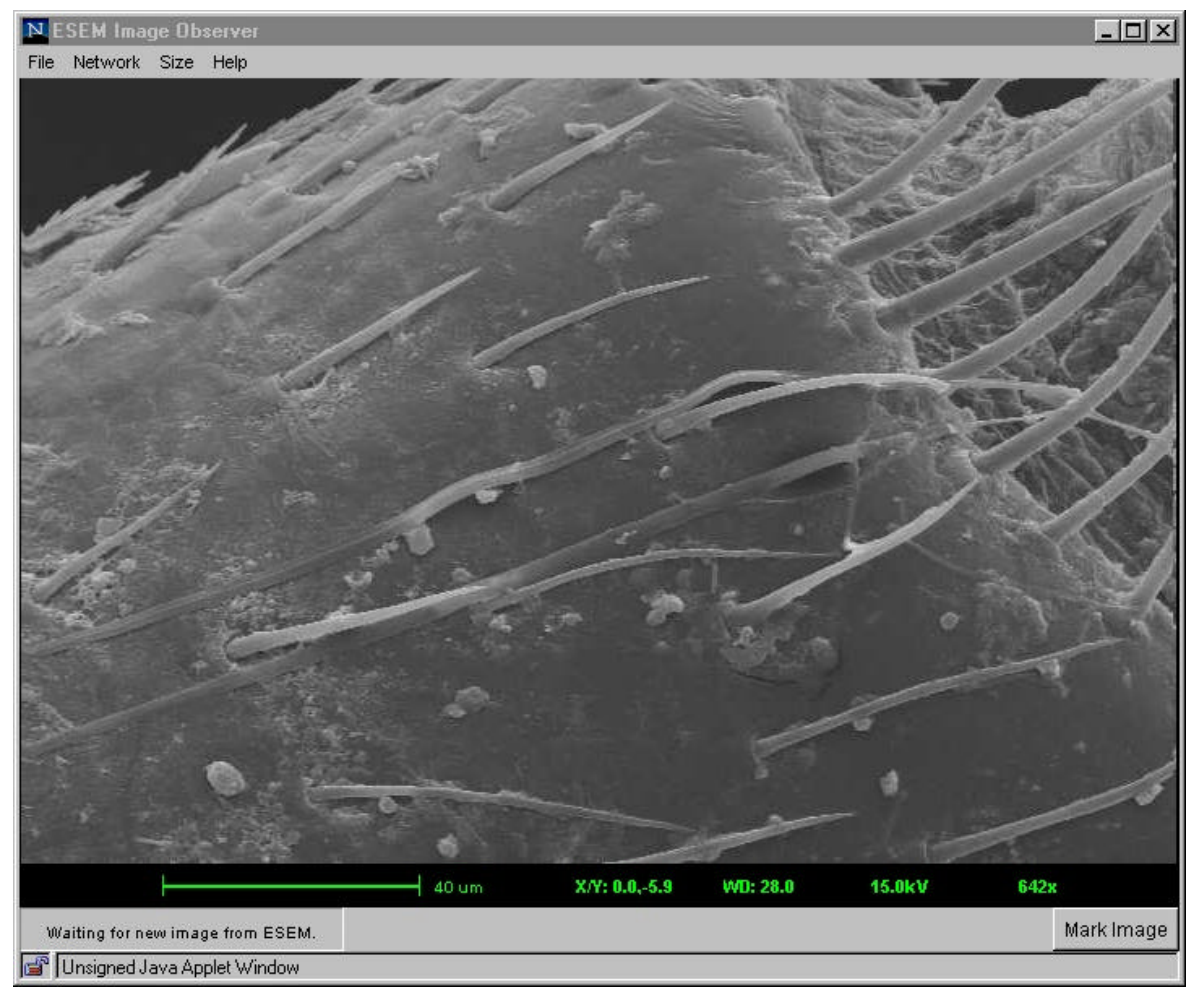

Figure 1 The ESEM Observer component of Bugscope. Anyone using a web browser connected to the Internet can observe a Bugscope session by visiting the project web page and loading the Observer.

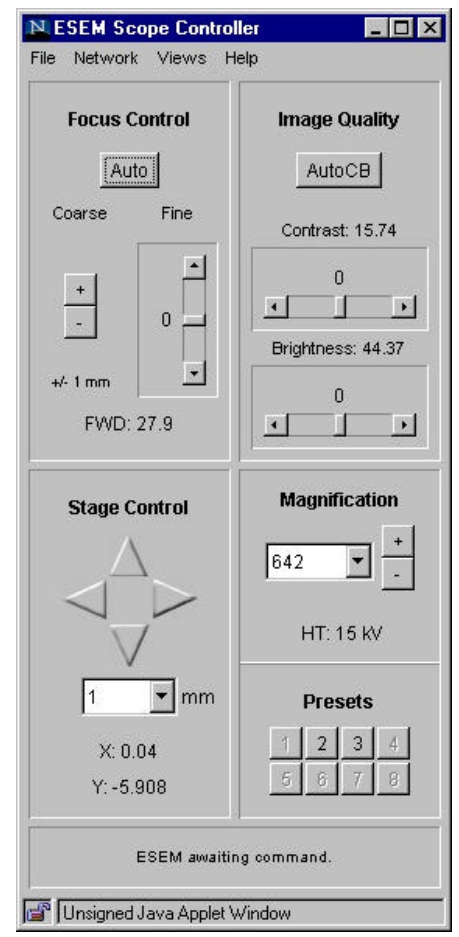

Figure 2 The ESEM Controller component. As you can see in this image of the Controller component, several ESEM controls are available to the remote user. The current project is an effort to further enhance the usability of the Observer and Controller components, raising the level of functionality to as closely as possible match that of the same high-level research instrument used hands on, rather than remotely. 